\title{
ON THE UNIQUENESS OF A MINIMAL NORM REPRESENTATIVE OF AN OPERATOR IN THE COMMUTANT OF THE COMPRESSED SHIFT
}

\author{
CIPRIAN FOIAS AND ALLEN TANNENBAUM
}

(Communicated by John B. Conway)

\begin{abstract}
In this note we give a new criterion guaranteeing the uniqueness of a minimal norm representative of a bounded linear operator which commutes with a finite multiplicity shift. We moreover give examples which show that if the hypotheses of our theorem are violated then the minimal norm representative may not be unique.
\end{abstract}

Introduction. In this note we give a new sufficient condition guaranteeing the uniqueness of minimal norm representative of a bounded linear operator in the commutant of a contraction of class $C_{0}(n)$ (see (1.1) below for the precise definition). The results discussed here complement some previous work of the authors [5] on the classical Nehari problem. Before explaining more precisely our main theorem, it will first be necessary to set up some notation and make some definitions. Let $\mathcal{E}$ denote a finite dimensional normed complex vector space. Then $H^{p}(\mathcal{E})$ will denote the standard $p$ th Hardy space of $\mathcal{E}$-valued functions on the unit circle $D$ equipped with the norm $\|\cdot\|_{p}$ induced from the norm of $\mathcal{E}$ for $1 \leq p \leq \infty$.

Explicitly, if $\|\cdot\|_{\mathcal{E}}$ is the norm on $\mathcal{E}$, then

$$
\|h\|_{p}^{p}:=\frac{1}{2 \pi} \int_{0}^{2 \pi}\left\|h\left(e^{i \theta}\right)\right\|_{\mathcal{E}}^{p} d \theta
$$

for $1 \leq p<\infty$, and

$$
\|h\|_{\infty}=\operatorname{ess} \sup \left\{\left\|h\left(e^{i \theta}\right)\right\|_{\varepsilon}: 0 \leq \theta \leq 2 \pi\right\} .
$$

For $\mathcal{E}=\mathbf{C}$, we set $H^{p}:=H^{p}(\mathbf{C})$. Following [14], we now make the following crucial definition.

DEFINITION (1.1). Let $T$ be a contraction on a complex Hilbert space $H$. Then we say that $T$ is of class $C_{0}(n)$ if the defect operators of $T$ have rank $n(<\infty)$, and if there exists a nonzero function $u \in H^{\infty}$ such that $u(T)=0$. (We are using the functional calculus notation of [14] here.)

This implies that $T$ is the compression of shift of finite multiplicity to the orthogonal complement of an invariant subspace of the shift. Indeed the functional model for the operator $T$ has the form

$$
T \cong P_{H} S \mid H, \quad H \cong H^{2}\left(\mathbf{C}^{n}\right) \ominus \Omega H^{2}\left(\mathbf{C}^{n}\right)
$$

where $\Omega$ is inner, $S$ denotes the unilateral shift on $H^{2}\left(\mathbf{C}^{n}\right)$. and $P_{H}: H^{2}\left(\mathbf{C}^{n}\right) \rightarrow H$ denotes orthogonal projection. (We will identify $H$ with $H^{2}\left(\mathbf{C}^{n}\right) \ominus \Omega H^{2}\left(\mathbf{C}^{n}\right)$

Received by the editors July 3, 1986 and, in revised form, August 28, 1986.

1980 Mathematics Subject Classification (1985 Revision). Primary 47A20, 47A15.

Key words and phrases. Compressed shift, commutant, Hardy spaces. 
throughout this paper.) We can now state the following special case of the "commutant lifting theorem" ([12]; see also $[\mathbf{1 3}, \mathbf{1 4}])$.

THEOREM (1.2) (NotATION AS ABOVE). A bounded linear operator $A: H \rightarrow$ $H$ commutes with $T$ (i.e. $A T=T A$ ) if and only if there exists $W \in H^{\infty}\left(\mathbf{C}^{n \times n}\right)$ such that $A=P_{H} W \mid H$. Moreover in this case, we may find $\hat{W} \in H^{\infty}\left(\mathbf{C}^{n \times n}\right)$ with $A=P_{H} \hat{W} \mid H$ and such that $\|\hat{W}\|_{\infty}=\|A\|$. (Note that we are using the identifications of (1) in the statement of Theorem (1.2). We regard $W$ as an operator on $H^{2}\left(\mathbf{C}^{n}\right)$ via $(W g)(z):=W(z) g(z)$.)

Now such a function $\hat{W}$ as given in (1.2) is called a minimal norm representative of $A$ in the sense that $\hat{W}$ has minimal $H^{\infty}$-norm among all the functions $\{W \in$ $\left.H^{\infty}\left(\mathbf{C}^{n \times n}\right): A=P_{H} W \mid H\right\}$. Of course, there is no reason to expect that a minimal norm representative will be unique, and indeed for vector-valued functions $(n>1)$, it is usually the case that the representative will not be unique. This important issue of uniqueness has been studied in a number of places, e.g. in Adamjan, Arov and Krein [1, 2], Arsene, Ceausescu and Foias [3], and Sarason [12]. The contribution of the present work (see Theorem (2.1)) is to give a new simple and generally applicable criterion guaranteeing that some $\hat{W}$ will be unique.

We should note that our interest in studying the uniqueness of the minimal norm representative is not only motivated by purely mathematical reasons. Indeed this question is intimately related to certain important design methods (so-called " $H^{\infty}$ optimization theory") that have become pervasive in control engineering in the past few years. The uniqueness of $\hat{W}$ is equivalent to the uniqueness of a certain optimal controller solving a weighted sensitivity problem for a possibly infinite dimensional dynamical system. See $[\mathbf{5 - 1 0}, \mathbf{1 5}, \mathbf{1 6}]$ for details. We should add that Theorem $(2.1)$ taken in conjunction with the authors' results in [5], gives a fairly comprehensive design procedure for the broadest class of systems of current interest in control theory.

Finally in $§ 3$, we will give some examples which show that violation of the hypotheses of Theorem (2.1) can result in a minimal norm representative being nonunique even in the scalar case $(n=1)$.

2. The uniqueness of the minimal norm representative. In this section we formulate and prove our main result about the uniqueness of the minimal norm representative of an operator commuting with an operator of class $C_{0}(n)$. Before doing this however, we will have to introduce some additonal notation.

For a given bounded linear operator $A$ on the Hilbert space $H, \sigma_{\text {ess }}(A)$ will denote the essential spectrum, and

$$
\rho_{\mathrm{ess}}(A):=\sup \left\{|\lambda|: \lambda \in \sigma_{\mathrm{ess}}(A)\right\} .
$$

the essential spectral radius. Let $w \in H^{\infty}$ be a scalar-valued function. Let $T$ be a contraction of class $C_{0}(n)$ on $H$, and let $I$ denote the $n \times n$ identity matrix. Then making the identifications of (1) of the Introduction, we set $w(T)=P_{H}(w I) \mid H$ where we regard $w I$ as an operator on $H^{2}\left(\mathbf{C}^{n}\right)$ in the obvious way. This agrees with the functional calculus notation of [14].

We are now ready to state our main result. 
THEOREM (2.1) (NOTATION AS ABOVE). Let $w \in H^{\infty}$, and suppose that $w$ is continuous on $\sigma_{\mathrm{ess}}(T)$. Set $A=W(T)$. If $\|A\|>\rho_{\mathrm{ess}}:=\rho_{\mathrm{ess}}(A)$, then the norm of $A$ is attained, and for a minimal norm representative $W \in H^{\infty}\left(C^{n \times n}\right)$ one has $\left\|W\left(e^{i \theta}\right)\right\|=\|A\|$ a.e. Moreover, in this case if a minimal norm representative is of the form $w_{i} I$ where $w_{i} \in H^{\infty}$ (i.e. $w_{i}$ is scalar-valued) and $I$ is the $n \times n$ identity, then $w_{i}$ is unique.

Proof. Without loss of generality we may assume that $\|A\|=1$. Let $\hat{W}$ be a minimal norm representative of $A$. Then by definition, $A=P_{H} \hat{W} \mid H$, and $\|\hat{W}\|_{\infty}=\|A\|=1$.

In this case, there exists a sequence $h_{j} \in H,\left\|h_{j}\right\|=1$ such that $\left\|A h_{j}\right\| \rightarrow 1$ as $j \rightarrow \infty$. Notice that

$$
\begin{aligned}
0 & \leq \frac{1}{2 \pi} \int_{0}^{2 \pi}\left\langle h_{j}\left(e^{i \theta}\right),\left(I-\hat{W}\left(e^{i \theta}\right)^{*} \hat{W}\left(e^{i \theta}\right) h_{j}\left(w^{i \theta}\right)\right\rangle d \theta\right. \\
& =1-\left\|\hat{W} h_{j}\right\|^{2} \leq 1-\left\|\left(P_{H} \hat{W}\right) h_{j}\right\|^{2}=1-\left\|A h_{j}\right\|^{2} \rightarrow 0
\end{aligned}
$$

as $j \rightarrow \infty$. (We are denoting the standard inner product on $\mathbf{C}^{n}$ by $\langle$,$\rangle and the$ Hilbert space norm on $H^{2}\left(\mathbf{C}^{n}\right)$ and $H$ by $\|\cdot\|$.)

Next by a theorem of Foias and Mlak [4] (see also [11]), we have that

$$
\sigma_{\text {ess }}(A)=\left\{w(\lambda): \lambda \in \sigma_{\text {ess }}(T)\right\} .
$$

(Note that we have assumed that $w \in H^{\infty}$ and $w$ is continuous on $\sigma_{\text {ess }}(T)$.)

We claim

$$
\partial D \not \subset \sigma_{\mathrm{ess}}(T)
$$

( $\partial D$ denotes the unit circle.) Indeed suppose to the contrary that $\partial D=\sigma_{\text {ess }}(T)$. Then we would have that

$$
\begin{aligned}
\rho_{\mathrm{ess}} & =\sup \left\{|w(\lambda)|: \lambda \in \sigma_{\mathrm{ess}}(T)\right\}=\|W\|_{\infty} \\
& =\sup \{|w(\lambda)|: \lambda \in \partial D\},
\end{aligned}
$$

contrary to hypothesis, since $\|w\|_{\infty} \geq\|A\|>\rho_{\text {ess. }}$. Hence (3) is valid.

Now we may assume without loss of generality that $h_{j} \rightarrow h$ weakly in $H$. But then from Sz.-Nagy and Foias [14] (see Chapter VI, §4) if follows that $h_{j}(\lambda) \rightarrow h(\lambda)$ uniformly on compact subsets of some fixed open neighborhood of $D \cup\left(\partial D \backslash \sigma_{\mathrm{ess}}(T)\right)$.

Let $\varepsilon>0, \varepsilon<<1$, and set

$$
\sigma_{\varepsilon}:=\left\{\theta \in[0,2 \pi]: I-\hat{W}\left(e^{i \theta}\right)^{*} \hat{W}\left(e^{i \theta}\right) \geq \varepsilon^{2} I\right\}
$$

where $I$ is the $n \times n$ identity matrix. From (2), we see that

$$
\int_{\sigma_{\varepsilon}}\left\langle h_{j}\left(e^{i \theta}\right), h_{j}\left(e^{i \theta}\right)\right\rangle d \theta \rightarrow 0 \quad \text { as } j \rightarrow \infty
$$

Thus from the above, we have that for any function $k \in H^{2}\left(\mathbf{C}^{n}\right)$,

$$
\frac{1}{2 \pi} \int_{0}^{2 \pi}\left\langle h_{j}\left(e^{i \theta}\right), k\left(e^{i \theta}\right)\right\rangle d \theta \rightarrow \frac{1}{2 \pi} \int_{0}^{2 \pi}\left\langle h\left(e^{i \theta}\right), k\left(e^{i \theta}\right)\right\rangle d \theta
$$

as $j \rightarrow \infty$, and

$$
\left|\frac{1}{2 \pi} \int_{0}^{2 \pi}\left\langle h\left(e^{i \theta}\right), k\left(e^{i \theta}\right)\right\rangle d \theta\right|^{2} \leq \frac{1}{2 \pi} \int_{[0,2 \pi] \backslash \sigma_{\varepsilon}}\left\|k\left(e^{i \theta}\right)\right\|^{2} d \theta .
$$


Clearly (5) implies that $h\left(e^{i \theta}\right)=0$ almost everywhere on $\sigma_{\varepsilon}$. We now claim that $h \neq 0$. Note that once we have proven this claim, we are done. Indeed certainly if $h \neq 0$, then the norm of $A$ is attained, i.e. $\|A h\|=\|A\|$. Moreover if $h \neq 0$, then $\sigma_{\varepsilon}$ has measure zero for every sufficiently small $\varepsilon>0$, and this means that $\left\|\hat{W}\left(e^{i \theta}\right)\right\|=1$, a.e. But then it is easy to see in this case that if $\hat{W}=w_{1} I$ with $w_{1} \in H^{\infty}$, this $w_{1}$ must be unique.

So we must prove now that $h \neq 0$. Suppose to the contrary $h=0$. Then let $B_{\delta}$ be the ball on $\partial D$ centered on $\sigma_{\text {ess }}(T)$ of radius $\delta$. Clearly $h_{j}\left(e^{i \theta}\right) \rightarrow 0($ as $j \rightarrow \infty)$ uniformly on $\partial D \backslash B_{\delta}$. Hence

$$
\begin{aligned}
1 \leftarrow & \left\|A h_{j}\right\|^{2} \leq\left\|w h_{j}\right\|^{2} \\
= & \frac{1}{2 \pi} \int_{\left\{e^{i \theta} \in B_{\delta}\right\}}\left|w\left(e^{i \theta}\right)\right|^{2}\left\langle h_{j}\left(e^{i \theta}\right), h_{j}\left(e^{i \theta}\right)\right\rangle d \theta \\
& +\frac{1}{2 \pi} \int_{\left\{e^{i \theta} \notin B_{\delta}\right\}}\left|w\left(e^{i \theta}\right)\right|^{2}\left|h_{j}\left(e^{i \theta}\right)\right|^{2} d \theta \\
\leq & \sup _{e^{i \theta} \in B_{\delta}}\left|w\left(e^{e \theta}\right)\right|^{2}+\frac{1}{2 \pi} \int_{\left\{e^{i \theta} \notin B_{\delta}\right\}}\left|w\left(e^{e \theta}\right)\right|^{2}\left\langle h_{j}\left(e^{i \theta}\right), h_{j}\left(e^{i \theta}\right)\right\rangle d \theta \\
\rightarrow & \sup _{e^{i \theta} \in B_{\delta}}\left|w\left(e^{i \theta}\right)\right|^{2} \quad \text { as } j \rightarrow \infty .
\end{aligned}
$$

But this implies

$$
1 \leq \sup _{e^{i \theta} \in B_{\delta}}\left|w\left(e^{i \theta}\right)\right|^{2} .
$$

On the other hand, we clearly have that

$$
\sup _{e^{i \theta} \in B_{\delta}}\left|w\left(e^{i \theta}\right)\right|^{2} \rightarrow \rho_{\mathrm{ess}}\left(w(T)^{2}\right)=\rho_{\mathrm{ess}}\left(A^{2}\right)<1
$$

as $\delta \rightarrow 0$. (Note that $\rho_{\text {ess }}\left(A^{2}\right)<1$ since by hypothesis $\rho_{\text {ess }}(A)<\|A\|=1$.) Hence from (6) and (7) we have arrived at a contradiction proving our claim that $h \neq 0$, and so the proof that the norm of $\|A\|$ is attained and that $\left\|W\left(e^{i \theta}\right)\right\|=\|A\|$ a.e. is complete.

Finally the uniqueness statement follows now from the fact that if $w_{1} I$ is a minimal norm representative for $A$ then by virtue of the already proven result, $w_{1} /\|A\|$ need be an inner function in $H^{\infty}$.

REMARKS (2.2). (i) Note that in the scalar case (when $n=1$ ), the above argument shows that if $\|w(T)\|>\rho_{\text {ess }}(w(T))$, then a minimal norm representative is unique.

(ii) When $w \in H^{\infty}$ is rational, and for $T$ the compressed shift on $H^{2} \ominus m H^{2}$ ( $m \in$ $H^{\infty}$ inner and nonconstant), the authors have in [5] devised a method for explicitly computing $\|w(T)\|$.

3. Some examples. In this section, we will take $T$ to be the compressed shift on $H^{2} \ominus m H^{2}$ where $m \in H^{\infty}$ is a nonconstant inner function. We will give two examples in order to illustrate the point that even in the scalar case, if the hypotheses of Theorem (2.1) are violated, then a minimal norm representative may not be unique. See also Sarason [12] for an example of a nonunique representative function. All the functions in this section are scalar-valued. 
EXAMPLE (3.1). In this first example we violate the hypothesis that $\|w(T)\|>$ $\rho_{\text {ess }}$ of (2.1). Explicitly, let

$$
\begin{gathered}
m(z):=\exp \left(\frac{z+1}{z-1}\right), \quad w_{1}(z):=\frac{1+z}{2} \\
w_{2}(z):=w_{1}(z)+\frac{1}{8} m(z)(1-z)^{2} .
\end{gathered}
$$

Clearly $\left\|w_{1}\right\|_{\infty}=1, w_{1}(T)=w_{2}(T)$, and $\left\|w_{1}(T)\right\|=1=\rho_{\text {ess }}\left(w_{1}(T)\right)$. Moreover $w_{1}$ and $w_{2}$ are continuous on $\sigma_{\text {ess }}(T)=\{1\}$.

Now we claim $\left\|w_{2}\right\|_{\infty}=1$. To see this note

$$
\left|w_{2}\left(e^{i \theta}\right)\right| \leq\left|\frac{1+e^{i \theta}}{2}\right|+\frac{1}{8}\left|1-e^{i \theta}\right|^{2}=\left|\cos \left(\frac{\theta}{2}\right)\right|+\frac{1}{2}\left(\sin \left(\frac{\theta}{2}\right)\right)^{2}
$$

for $0 \leq \theta \leq 2 \pi$. But this implies

$$
\left\|w_{2}\right\|_{\infty}=\sup _{\theta \in[0,2 \pi]}\left|w_{2}\left(e^{i \theta}\right)\right|=\left|w_{2}(1)\right|=1
$$

as claimed.

Hence we have that

$$
\left\|w_{1}\right\|_{\infty}=\left\|w_{1}(T)\right\|=\left\|w_{2}(T)\right\|=\left\|w_{2}\right\|_{\infty}=1,
$$

and so both $w_{1}$ and $w_{2}$ are minimal norm representatives, i.e. we do not have uniqueness.

EXAMPLE (3.2). In this example we violate the hypothesis of (2.1) that $w$ is continuous on the essential spectrum of the compressed shift. Again we let $m(z)$, $w_{1}(z), w_{2}(z)$ be as in Example (3.1).

Let $\hat{T}$ denote the compression of the shift to $H^{2} \ominus m^{2} H^{2}$. ( $T$ denotes the compression of the shift to $H^{2} \ominus m H^{2}$.) Now set

$$
A:=\left(m w_{1}\right)(\hat{T}) \text {. }
$$

Then

$$
\left(m w_{2}\right)(\hat{T})-\left(m w_{1}\right)(\hat{T})=\frac{1}{8} m^{2}(\hat{T})(I-\hat{T})^{2}=0
$$

and so

$$
A=\left(m w_{1}\right)(\hat{T})=\left(m w_{2}\right)(\hat{T})
$$

Moreover

$$
A^{2}=\left(m^{2} w_{1}^{2}\right)(\hat{T})=m^{2}(\hat{T}) w_{1}^{2}(\hat{T})=0
$$

which implies that $\rho_{\text {ess }}(A)=0$, and so $\|A\|>\rho_{\text {ess }}(A)$. Note that we have the expression

$$
A=w_{1}(\hat{T}) m(\hat{T})
$$

Now set

$$
\not 1:=\left(m(\hat{T})\left(H^{2} \ominus m^{2} H^{2}\right)\right)^{-} \text {. }
$$

We claim that $\hat{T} \mid \mathcal{H}$ is unitarily equivalent to $T$. Indeed it is easy to see that

$$
V:=m(\hat{T}) \mid H^{2} \ominus m H^{2}
$$

is unitary from $H^{2} \ominus m H^{2} \rightarrow \mathcal{H}$ and $\hat{T} V=V T$. Hence we get that

$$
A\left|H^{2} \ominus m H^{2}=w_{1}(\hat{T}) m(\hat{T})\right| H^{2} \ominus m H^{2}=w_{1}(\hat{T}) V=V w_{1}(T) .
$$


It then follows that

$$
\left\|A \mid H^{2} \ominus m H^{2}\right\|=\left\|w_{j}(T)\right\|=\left\|w_{j}\right\|_{\infty}=1
$$

for $j=1$, 2. But $\|A\| \leq\left\|m w_{1}\right\|_{\infty}=\left\|m w_{2}\right\|_{\infty}=1$, and so $\|A\|=1$.

In other words, we have shown that $\left\|m w_{j}\right\|_{\infty}=\left\|\left(m w_{j}\right)(\hat{T})\right\|=1$ for $j=1,2$. This means of course that both $m w_{1}$ and $m w_{2}$ are minimal norm representatives for our given $A$, and once again we do not have uniqueness.

REMARK (3.3). This case in which $m(z)=\exp ((z+1) /(z-1))$ is very important in the control of delay systems. One can prove (see [6] for details) that for rational $w \in H^{\infty}$ with the property that $|w|$ attains its absolute minimum at $z=1$, the minimal norm representative is unique. System-theoretically this means that the $H^{\infty}$-optimal compensator for a delay system relative to a low-pass filter is unique. See [5-9] for a more complete discussion.

\section{REFERENCES}

1. V. M. Adamjan, D. Z. Arov, and M. G. Krein, Infinite Hankel matrices and generalized Carathéodory-Fejér and I. Schur problems, Funct. Anal. Appl. 2 (1968), 169-181.

2. __ Analytic properties of Schmidt pairs for a Hankel operator and the generalized Schur-Takagi problem, Math. USSR Sb. 15 (1971), 31-73.

3. Gr. Arsene, Z. Ceausescu, and C. Foias, On intertwining dilations. VIII, J. Operator Theory 4 (1980), 55-91.

4. C. Foias and W. Mlak, The extended spectrum of completely non-unitary contractins and the spectral mapping theorem. Studia Math. 26 (1966), 239-245.

5. C. Foias and A. Tannenbaum, On the Nehari problem for a certain class of $L^{\infty}$-functions appearing in control theory, Technical Report, Department of Electrical Engineering, McGill University, February 1986; J. Funct. Anal. (to appear).

6. C. Foias, A. Tannenbaum, and G. Zames, Sensitivity minimization for arbitrary SISO distributed plants, Systems and Control Lett. 8 (1987), 189-195.

7. Weighted sensitivity minimization for delay systems, IEEE Trans. Automatic Control AC-31 (1986), 763-766.

8. 25 (1987), 686-705.

9. Lett. 7 (1986), 239-246.

10. B. A. Francis and J. Doyle, Linear control theory with an $H^{\infty}$ optimality criterion, SIAM J. Control Optim. (to appear).

11. N. K. Nikolskii, Treatise on the shift operator, Springer-Verlag, Berlin, New York and 1985.

12. D. Sarason, Generalized interpolation in $H^{\infty}$, Trans. Amer. Math. Soc. 127 (1967), 179-203.

13. B. Sz.-Nagy and C. Foias, Dilation des commutants d'opérateurs, C. R. Acad. Sci. Paris Sér. A 266 (1967), 493-495.

14. __ Harmonic analysis of operators on Hilbert space, North-Holland, Amsterdam, 1970.

15. A. Tannenbaum, Invariance and system theory: Algebraic and geometric aspects, Lecture Notes in Math., vol. 845, Springer-Verlag, Berlin and New York, 1981.

16. G. Zames, Feedback and optimal sensitivity: model reference transformations, seminorms, and approximate inverses, IEEE Trans. Automatic Control AC-23 (1981), 301-320. 47405

Department of MAThematics, Indiana University, Bloomington, Indiana

Department of Electrical Engineering, University of Minnesota, MinNEAPOLIS, MINNESOTA 55455

Department of MAthematics, Ben-Gurion University, Beer SheVA, IsRael 